\title{
Curcumin increases cholesterol efflux via heme oxygenase-1-mediated ABCA1 and SR-BI expression in macrophages
}

\author{
YI ZHONG, JIAN FENG, ZHONGCAI FAN and JIAFU LI
}

Department of Cardiology, The Affiliated Hospital of Southwest Medical University, Luzhou, Sichuan 646000, P.R. China

Received March 6, 2017; Accepted November 6, 2017

DOI: $10.3892 / \mathrm{mmr} .2018 .8577$

\begin{abstract}
Curcumin, which is an extract from a traditional Chinese medicine, has previously been demonstrated to exhibit an anti-atherosclerotic effect, which is closely associated with an increase in cholesterol efflux. However, it is unclear as to whether the increased effect is mediated by heme oxygenase (HO)-1. Macrophages were treated with different concentrations of curcumin, HO-1 inhibitor and small interfering (si)RNA in different experiments. Analysis of protein expression was conducted via western blotting. mRNA expression levels were measured using reverse transcription-polymerase chain reaction. Antioxidant response element (ARE)-driven promoter activity was measured by a dual-luciferase reporter assay. The cholesterol efflux analysis was performed by fluorescence-labelled cholesterol (NBD) using a multi-label counter. In the present study, the results indicated that curcumin increased the cholesterol efflux from macrophages. Additionally, curcumin significantly upregulated HO-1 expression. The HO-1 inhibitor (zinc protoporphyrin) partly blocked this effect. Curcumin also promoted scavenger receptor class B type I (SR-BI) and ATP-binding cassette transporter A1 (ABCA1) expression. HO-1 small interfering (si)RNA partly abolished the increased SR-BI and ABCA1 expression induced by curcumin. Furthermore, the nuclear factor, erythroid 2 like 2 (Nrf2) expression in the nucleus was dose-dependently increased by curcumin. Nrf2 siRNA successfully inhibited the curcumin-induced HO-1 expression. Curcumin significantly increased Nrf2-driven luciferase activity. Overall, these data indicated that curcumin activates the Nrf2-ARE signaling pathway and upregulates HO-1 expression, which mediates SR-BI and ABCA1 expression and thereby increases cholesterol efflux.
\end{abstract}

Correspondence to: Dr Yi Zhong, Department of Cardiology, The Affiliated Hospital of Southwest Medical University, 25 Tai Ping Street, Luzhou, Sichuan 646000, P.R. China

E-mail: zhongyi1982529@163.com

Key words: curcumin, cholesterol efflux, heme oxygenase-1, ATP-binding cassette transporter A1, scavenger receptor class B type I

\section{Introduction}

It has previously been demonstrated that foam cell formation occurs when monocyte-derived macrophages accumulate atherogenic lipoproteins, and is one of the hallmarks of early atherosclerosis $(1,2)$. Vascular smooth muscle cell migration to the vascular intima and pro-inflammatory cytokine secretion are important steps in the development of atherosclerosis $(3,4)$. The foam cells derived from macrophages are primarily formed by the excessive uptake of oxidative low density lipoprotein $(5,6)$. The reverse cholesterol transporters (RCTs) include ATP-binding cassette transporter (ABC) A1, ABCG1 and scavenger receptor class B type I (SR-BI), which mediate the intracellular cholesterol efflux to high density lipoprotein (HDL) or apolipoprotein (apo) A-I (7,8). Ample evidence indicates that increased RCT expression through anti-inflammatory and antioxidant activity may reduce the accumulation of intracellular cholesterol, thereby inhibiting the progression of atherosclerosis $(9,10)$.

Curcumin, extracted from Curcuma longa, a widely used traditional Chinese herb for the treatment of human diseases, has been reported to exhibit several beneficial effects in the cardiovascular system, including anti-inflammatory and anti-oxidant $(11,12)$. Currently, there is little information regarding the role of curcumin in the setting of cholesterol efflux. Additionally, accumulating evidence indicates that heme oxygenase-1 (HO-1) contributes to the effect of anti-inflammation and anti-oxidation in several cell types and animal models (13-16). However, it remains unclear whether HO-1 is involved in the promoting effect of curcumin on cholesterol efflux. If it were identified that HO-1 was involved in the promoting effect of curcumin on cholesterol efflux, then whether the reverse cholesterol transporters (ABCA1, ABCG1 and SR-BI) serve an important role is worthy of further study.

The present study aimed to explore the effect of curcumin on cholesterol efflux in macrophages. It was demonstrated that curcumin enhances SR-BI and ABCA1 expression. It was additionally observed that curcumin activated nuclear factor, erythroid 2 like (Nrf) 2-antioxidant response element (ARE) and upregulated the expression of HO-1, which mediated ABCA1 and SR-BI expression, and thereby promoted cholesterol efflux. 


\section{Materials and methods}

Cell culture and treatment. RAW264.7 macrophage cells (American Type Culture Collection, Manassas, VA, USA) were cultured in Dulbecco's modified Eagle's medium (Gibco; Thermo Fisher Scientific, Inc., Waltham, MA, USA) supplemented with $10 \%$ fetal bovine serum (Gibco; Thermo Fisher Scientific, Inc.), $100 \mathrm{U} / \mathrm{ml}$ penicillin and $100 \mu \mathrm{g} / \mathrm{ml}$ streptomycin at $37^{\circ} \mathrm{C}$ in a humidified atmosphere containing $5 \% \mathrm{CO}_{2}$. THP-1 cells (American Type Culture Collection) were cultured in RPMI-1640 (Gibco; Thermo Fisher Scientific, Inc.) and underwent induced differentiation into macrophages, as previously described (17). Cells were cultured in serum-free medium for $24 \mathrm{~h}$, and treated with curcumin (Sigma-Aldrich; Merck KGaA, Darmstadt, Germany) or zinc protoporphyrin (ZnPP; Sigma-Aldrich; Merck $\mathrm{KGaA}$ ) for $1 \mathrm{~h}$ at $37^{\circ} \mathrm{C}$. The purity of curcumin (Sigma-Aldrich; Merck KGaA) was $99 \%$ and the different concentrations of curcumin solution were configured by PBS.

Preparation of nuclear proteins. Nuclear protein was extracted from cells as previously described (18). Protein content was determined using a bicinchoninic assay protein reagent (Beyotime Institute of Biotechnology, Haimen, China).

Reverse transcription-polymerase chain reaction (RT-PCR). Total RNA was extracted using TRIzol ${ }^{\circledR}$-reagent (Invitrogen; Thermo Fisher Scientific, Inc.). RT-PCR was performed by using Invitrogen One step III ${ }^{\mathrm{TM}}$ Reverse Transcription PCR kit (Invitrogen; Thermo Fisher Scientific, Inc.) according to the manufacturer's protocol. The PCR thermocycling conditions were as follows: 30 cycles of $30 \mathrm{sec}$ at $93^{\circ} \mathrm{C}$ for denaturation, $30 \mathrm{sec}$ at $56^{\circ} \mathrm{C}$ for annealing, $45 \mathrm{sec}$ at $73^{\circ} \mathrm{C}$ for extension. Primer sequences: Forward, $5^{\prime}$-GGGTGACAGAAGAGGCTA AGACC-3' and reverse, 5'-AGATTCTCCCCTGCAGAGAGA AG-3' for HO-1; GAPDH forward, 5'-TGCACCACCAAC TGCTTAGC-3' and reverse, 5'-GGCATGGACTGTGGTCAT GA-3'. The products were analyzed in $1.5 \%$ agarose gel electrophoresis, stained with ethidium bromide (Sigma-Aldrich; Merck KGaA) and images were then captured under ultraviolet light. GAPDH served as the internal control.

Western blot analysis. Proteins were extracted from cells using radioimmunoprecipitation lysis buffer (Santa Cruz Biotechnology, Inc., Dallas, TX, USA) for $30 \mathrm{~min}$ at $4^{\circ} \mathrm{C}$. The protein content was determined using a Bicinchoninic Acid Protein Assay kit (Sigma-Aldrich; Merck KGaA). Total proteins (50 $\mu \mathrm{g}$ per lane) were separated by $10 \%$ SDS-PAGE and transferred onto nitrocellulose membranes. Membranes were blocked at room temperature for $1 \mathrm{~h}$ with $8 \%$ skimmed milk in Tris-buffered saline, followed by overnight incubation at $4^{\circ} \mathrm{C}$ with primary antibodies and then washed, prior to incubation for $2 \mathrm{~h}$ at room temperature with a goat anti-rabbit horseradish peroxidase-conjugated $\mathrm{IgG}$ secondary antibody (cat. no. SAB5300163; 1:1,000; Sigma-Aldrich; Merck KGaA) secondary antibody staining. The rabbit primary antibodies (Anti-ABCA1 cat. no. ab18180; 1:2,000; ABCG1; cat. no. ab52617; 1:2,000 and SR-BI; cat. no. ab52629; 1:2,000) were purchased from Abcam (Cambridge, UK). The other antibodies were purchased from Beyotime Institute of Biotechnology. Anti-HO-1 (cat. no. AF1333; 1:2,000) and
Anti-Nrf2 (cat. no. AF1609; 1:3,000). The bands were visualized using an Enhanced Chemiluminescence system (GE Healthcare Life Sciences, Little Chalfont, UK), and the band density was determined by Image $\mathbf{J}$ software version 1.38 (National Institutes of Health, Bethesda, MD, USA).

siRNA transfection. Macrophages were transfected with Nrf2 siRNA, or HO-1 siRNA using Lipofectamine 2000 (Invitrogen; Thermo Fisher Scientific, Inc.) for $24 \mathrm{~h}$. Following transfection for $24 \mathrm{~h}$, the cells were treated with curcumin for another $12 \mathrm{~h}$, then lysed for western blot analysis.

Transient transfection and luciferase assay. Cells were seeded in 24-well plates and grown to $70 \%$ confluence. Cells were transfected with pGL-ARE and pRL-TK plasmid (provided by Dr Li, Southern Medical University, Guangdong, China) using Lipofectamine ${ }^{\circledR} 2000$ (Invitrogen; Thermo Fisher Scientific, Inc.) according to the manufacturer's protocol. Following transfection for $24 \mathrm{~h}$, the cells were treated with different concentrations of curcumin $(10,20,40 \mu \mathrm{M})$ for $6 \mathrm{~h}$. Dual luciferase reporter assay was performed on the lysed cells co-transfected with pGL-ARE (firefly luciferase) and pRL-TK (Renilla luciferase). ARE-driven promoter activity was analyzed by a dual-luciferase reporter assay system (Dual-Glo ${ }^{\circledR}$ Luciferase Assay System, Promega Corporation, Madison, WI, USA). ARE luciferase activity was normalized to the Renilla luciferase activity in each sample.

Cholesterol efflux assay. Cholesterol efflux was determined as previously described (10). Cells (treated with curcumin) were equilibrated with NBD cholesterol (Sigma-Aldrich; Merck KGaA; $1 \mu \mathrm{g} / \mathrm{ml})$ in the presence of apoAI $(10 \mu \mathrm{g} / \mathrm{ml})$. Cells with NBD cholesterol labeling were washed with PBS and incubated for $6 \mathrm{~h}$ with RPMI-1640 medium. Cholesterol from cells released into the medium with fluorescent label was tested by a multilabel counter with $485 \mathrm{~nm}$ excitation and $535 \mathrm{~nm}$ emission.

Statistical analysis. All statistical analyses were performed using SPSS software version 13.0 (SPSS, Inc., Chicago, IL, USA). All the data were presented as the mean \pm standard deviation of three assays. Statistical analysis was performed using one-way analysis of variance followed by Tukey's post hoc test. $\mathrm{P}<0.05$ was considered to indicate a statistically significant difference.

\section{Results}

Curcumin promotes cholesterol efflux in Raw264.7 cells and THP-1 macrophages and ZnPP (HO-1 inhibitor) attenuates promoting effect. The present study investigated the effect of curcumin on cholesterol efflux. It was demonstrated that compared with control group, curcumin significantly promoted cholesterol efflux in a dose-dependent manner (10, 20 and $40 \mu \mathrm{M}$ ) in macrophages (Fig. 1A and B). In order to study whether HO-1 mediated this effect, cells were pre-treated with ZnPP. The results indicated that the effect was partly blocked by $\mathrm{ZnPP}$ (Fig. $1 \mathrm{C}$ and $\mathrm{D}$ ).

Curcumin increases HO-1 expression in a dose-dependent manner. The effect of curcumin on the expression of HO-1 
A

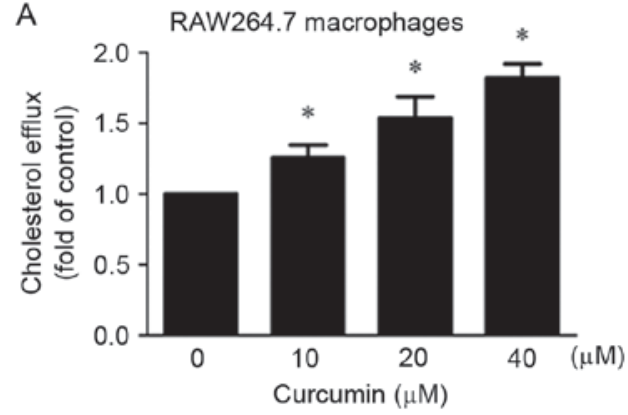

C

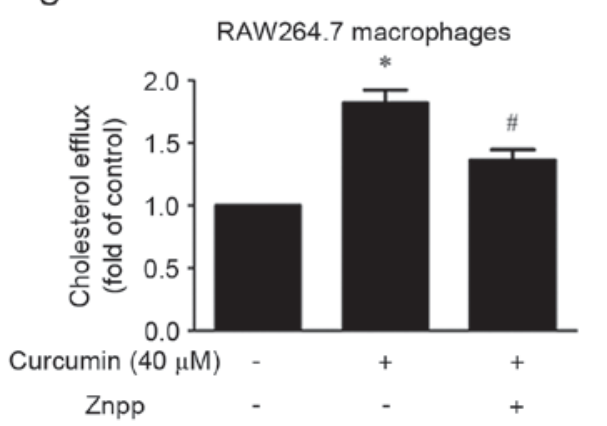

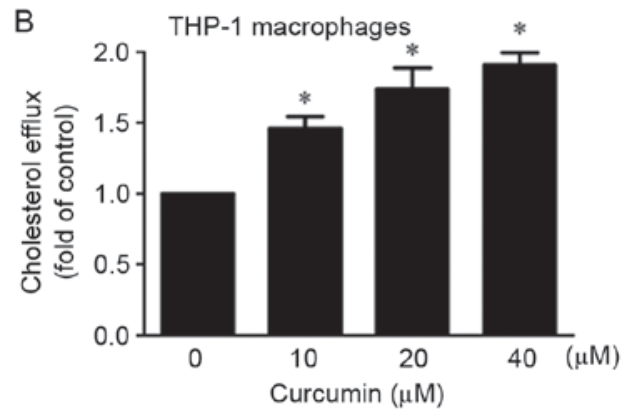

D
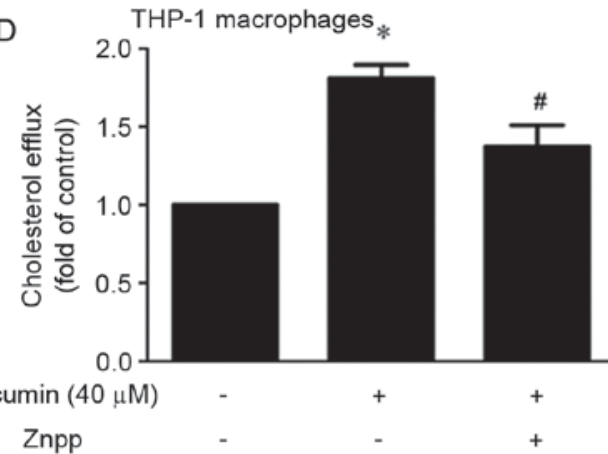

Figure 1. Curcumin promotes apolipoprotein A-I-mediated cholesterol efflux in Raw264.7 cells and THP-1 macrophages and ZnPP (HO-1 inhibitor) attenuates promoting effect of curcumin on cholesterol efflux. (A) Raw264.7 cells and (B) THP-1-derived macrophages were treated with indicated concentrations of curcumin for $12 \mathrm{~h}$, followed by NBD cholesterol $(1 \mathrm{mg} / \mathrm{ml})$ treatment for an additional $6 \mathrm{~h}$ in the presence of curcumin. (C) Raw264.7 cells and (D) THP-1-derived macrophages were pre-treated with $\mathrm{ZnPP}(10 \mu \mathrm{M})$ for 30 min and treated with indicated concentrations of curcumin for $12 \mathrm{~h}$, followed by NBD cholesterol $(1 \mathrm{mg} / \mathrm{ml})$ treatment for an additional $6 \mathrm{~h}$ in the presence of curcumin. Cholesterol efflux was expressed as a percentage of fluorescence in the medium relative to the total amount of fluorescence. ${ }^{*} \mathrm{P}<0.05$ vs. control group, ${ }^{\#} \mathrm{P}<0.05$ vs. curcumin-treated group. Data are expressed as the mean \pm standard deviation of three independent experiments. ZnPP, zinc protoporphyrin.

was detected by western blotting and RT-PCR in RAW264.7 macrophages. The results demonstrated that curcumin increased HO-1 expression at the mRNA and protein levels in RAW264.7 macrophages (Fig. 2).

Curcumin upregulates $A B C A 1$ and $S R-B I$ expression and HO-1 small interfering (si)RNA transfection attenuates the effect of curcumin on ABCAI and SR-BI expression. The results demonstrated that curcumin upregulates ABCA1 and SR-BI expression, and had no effect on expression of ABCG1 in RAW264.7 macrophages (Fig. 3A). In order to verify whether HO-1 mediated the effect of curcumin on ABCA1 and SR-BI expression, HO-1 siRNA (600 nM) was transfected into macrophages, and it was observed that the expression of HO-1 induced by curcumin decreased (Fig. 3B). Additionally, HO-1 siRNA transfection attenuated curcumin upregulation of the expression of ABCA1 and SR-BI. This result suggested that HO-1 exhibits a key role in regulating the expression of ABCA1 and SR-BI (Fig. 3C).

Nrf2-ARE pathway mediates curcumin-induced HO-I expression. Nrf2 has been verified to exhibit a key role in $\mathrm{HO}-1$ expression (19). As demonstrated in the results, Nrf2 expression in the nucleus was dose-dependently increased when cells were treated with curcumin (Fig. 4A). In addition, transfection with siRNA Nrf2 significantly inhibited the expression of HO-1 induced by curcumin (Fig. 4B). Next, RAW264.7 cells treated with curcumin were transfected with pGL-ARE and pRL-TK plasmids containing ARE promoter region and the

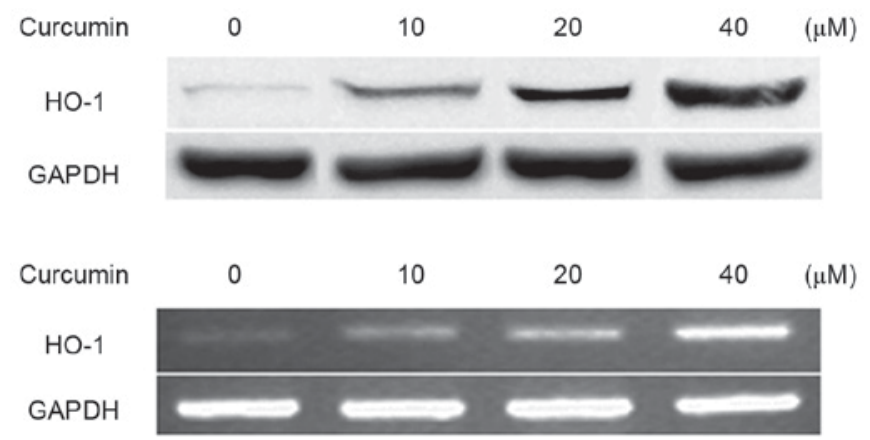

Figure 2. Curcumin significantly upregulates HO-1 mRNA and protein expression in a concentration-dependent manner. Macrophages were incubated with various concentrations of curcumin $(10,20$ and $40 \mu \mathrm{M})$ for $12 \mathrm{~h}$ and $\mathrm{HO}-1$ expression was determined by western blot analysis and reverse transcription-polymerase chain reaction. Results are representative of three independent experiments. HO-1, heme oxygenase-1.

luciferase structural gene. It was demonstrated that curcumin significantly elevated Nrf2-driven luciferase activity in a concentration-dependent manner (Fig. 4C).

\section{Discussion}

An important reason for the progression of atherosclerosis is that monocytes differentiate into macrophages which accumulate cholesterol in the blood vessel wall to form foam cells. HDL is an important lipoprotein for cholesterol 
A
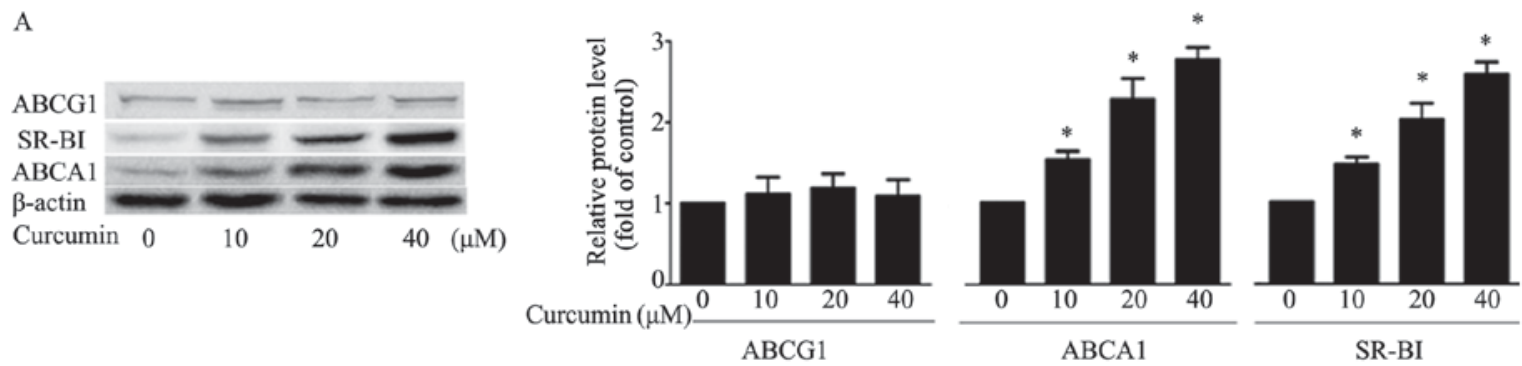

B

C
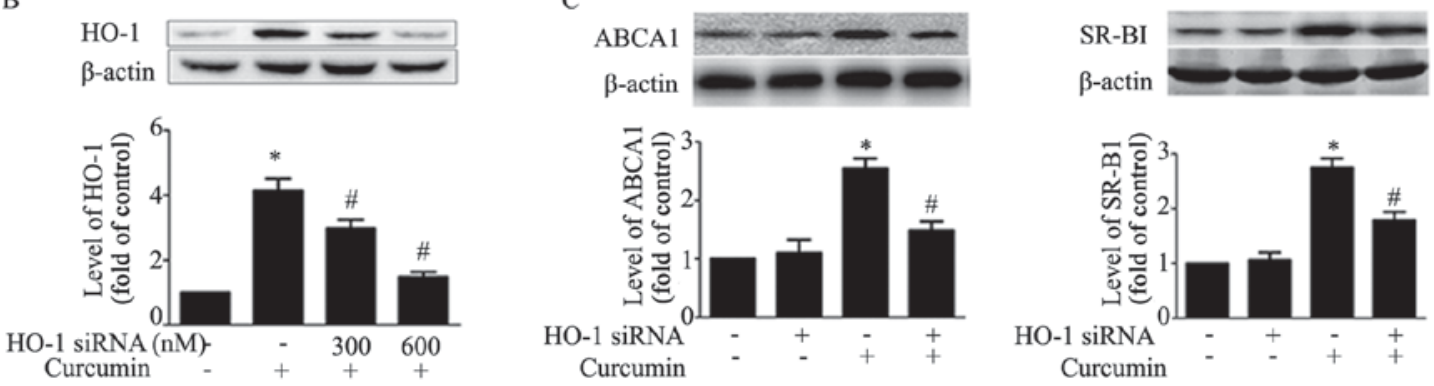

Figure 3. Effects of curcumin on protein expression of SR-BI, ABCA1, ABCG1 and HO-1 mediates this effect in macrophages. (A) Macrophages were treated with indicated concentrations $(0,10,20,40 \mu \mathrm{M})$ of curcumin for $12 \mathrm{~h}$ and the protein level of SR-BI, ABCA1 and ABCG1, or $\beta$-actin was determined by western blotting. " $\mathrm{P}<0.05$ vs. untreated group. (B) Macrophages were transfected with various concentrations of HO-1 siRNA ( $300,600 \mathrm{nM})$ for $24 \mathrm{~h}$, followed by curcumin treatment $(40 \mu \mathrm{M})$ for an additional $12 \mathrm{~h}$. Protein expression of HO-1 and $\beta$-actin was measured by western blotting. (C) Macrophages were pre-treated with HO-1 siRNA (600 nM) for $24 \mathrm{~h}$, followed by curcumin for an additional $12 \mathrm{~h}$. Protein levels of SR-BI, ABCA1 and $\beta$-actin was determined by western blotting. "P $<0.05$ vs. vehicle-treated group; ${ }^{\#} \mathrm{P}<0.05$ vs. curcumin alone group. Data are presented as the mean \pm standard deviation of three independent experiments. HO-1, heme oxygenase-1; si, small interfering; SR-BI, scavenger receptor class B type I; ABC, ATP-binding cassette transporter.

A
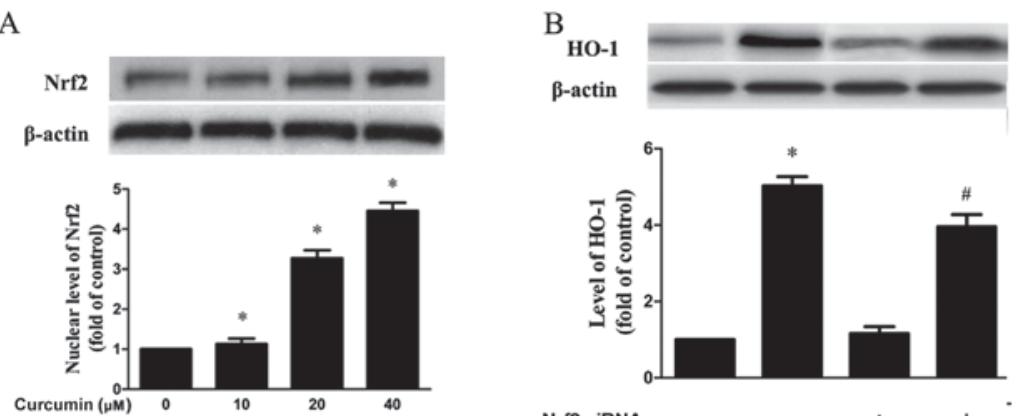

$\beta$-actin
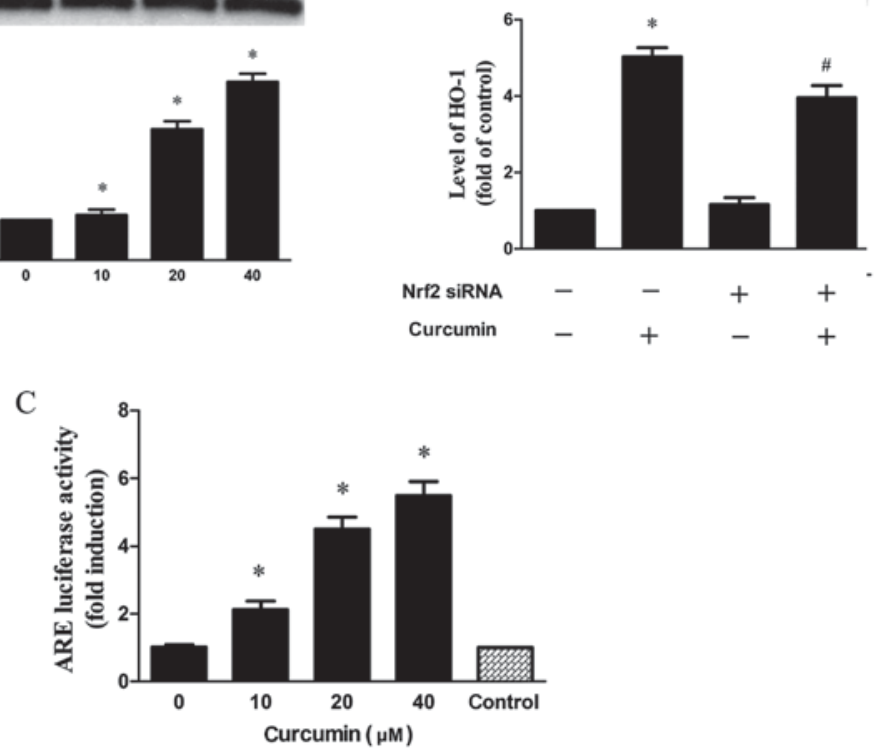

Figure 4. Nrf2-ARE is involved in the curcumin-mediated upregulation of HO-1. (A) Macrophages were incubated with indicated concentrations of curcumin for $60 \mathrm{~min}$ and nuclear extracts were subjected to western blotting to determine the level of Nrf2 and $\beta$-actin. (B) Following transfection of Nrf2 siRNA $(200 \mathrm{nM})$ for $24 \mathrm{~h}$, macrophages were treated with curcumin $(40 \mu \mathrm{M})$ for an additional $12 \mathrm{~h}$. The expression of HO-1 or $\beta$-actin was examined by western blotting. ${ }^{*} \mathrm{P}<0.05$ vs. untreated group; ${ }^{*} \mathrm{P}<0.05$ vs. curcumin alone group. (C) Cells were transiently transfected with pGL-ARE luciferase plasmid and pRL-TK for $24 \mathrm{~h}$, then treated with different concentrations of curcumin $(10,20,40 \mu \mathrm{M})$ for $6 \mathrm{~h}$. Dual luciferase reporter assay was performed on the lysed cells co-transfected with pGL-ARE (firefly luciferase) and pRL-TK (Renilla luciferase). ARE luciferase activity was normalized to the Renilla luciferase activity in each sample. Data are presented as the mean \pm standard deviation and are representative of at least 3 independent experiments; " $\mathrm{P}<0.05$ vs. control. si, small interfering; HO-1, heme oxygenase-1; Nrf2, nuclear factor, erythroid 2 like 2; ARE, antioxidant response element.

reverse transport, which is the process of removing cholesterol from the peripheral cells (20). It has previously been demonstrated that ABCA1, SR-BI and ABCG1 exhibit key roles in cholesterol efflux (7-9). Therefore, SR-BI, ABCA1 
and ABCG1 may be used as therapeutic targets to prevent and cure atherosclerosis.

It has been verified that curcumin has a protective effect in atherosclerosis (21). However, the underlying molecular and cellular mechanisms of the cholesterol metabolism have not been fully elucidated. The present study investigated the effect of curcumin on cholesterol efflux in macrophages. Firstly, it was demonstrated that curcumin promoted cholesterol efflux from macrophages, which was consistent with several previous reports $(22,23)$. HO-1 is an inducible antioxidant enzyme that mediates heme degradation to iron and carbon monoxide, which have therapeutic effects in atherosclerotic vascular diseases and inflammation (24). However, the role of HO-1 regarding curcumin promotion of cholesterol efflux is largely unknown. Therefore, cells were pre-treated with $\mathrm{ZnPP}$ (a HO-1 inhibitor), and it was observed that the curcumin-induced effect of increased cholesterol efflux was partially abolished. In addition, the results indicated that curcumin significantly increased HO-1 expression in a dose-dependent manner. Overall, the data suggested that increased HO-1 expression induced by curcumin partly contributed to the increased cholesterol efflux.

Data from previous studies suggest that HO-1-dependent mechanisms mediate the regulation of cholesterol homeostasis by other active agents (25-27). However, the effect of HO-1 on the expression of SR-BI and ABCA1 remains to be fully elucidated. The results of the present study demonstrated that curcumin increased SR-BI and ABCA1 expression in macrophages. In accordance with these results, it has been reported that curcumin upregulates SR-BI and ABCA1 expression in macrophages $(22,23)$. The results of the experiments additionally indicated that the deletion of the $\mathrm{HO}-1$ gene led to an inhibitory effect on the curcumin-induced increase in ABCA1 and SR-BI expression. The HO-1 gene may be activated by various factors at the transcription level, including Nrf2, nuclear factor- $\kappa \mathrm{B}$ and activator protein-1 (28). Of these factors, Nrf2 is important in upregulating HO-1 expression. Typically, Nrf2 and its inhibitor Kelch Like ECH Associated Protein (Keap)1 are located in the cytoplasm. Once stimulated, Nrf2 is separated from Keap1, and translocates to the nucleus, where it combines with ARE, leading to the promotion of the expression of HO-1 (29). Nuclear Nrf2 accumulation is a key step in the activation of the Nrf2-ARE pathway as a transcription factor (30).

In conclusion, the results indicated that curcumin enhanced Nrf2 expression in the nucleus. When cells were transfected with Nrf2 siRNA, curcumin-induced HO-1 expression was significantly reduced. Curcumin increased ARE luciferase activity in a concentration dependent manner, which suggested that curcumin exhibits the ability to induce transcription of different ARE associated genes. Overall, the results indicated that HO-1 participates in the upregulation of SR-BI and ABCA1 expression induced by curcumin via the Nrf2-ARE pathway in macrophages. The present study introduced a novel research direction for the key role of HO-1 in the curcumin-induced promotion of cholesterol efflux in macrophages. These results suggested that curcumin activated the Nrf2-ARE pathway and upregulated HO-1 expression, which mediated SR-BI and ABCA1 expression and thereby promoted cholesterol efflux. Therefore, the present study may provide a positive direction for research in atherosclerosis and it may provide a potential therapeutic molecular target for atherosclerosis. Further investigation is required to investigate various in vivo models in the future.

\section{Acknowledgements}

Not applicable.

\section{Funding}

The present study was partly supported by a research grant from science and technology strategic cooperation project of Luzhou Municipal People's Government-Luzhou Medical College (grant no. 2013LZLY-J53).

\section{Availability of data and materials}

The datasets used and/or analyzed during the current study are available from the corresponding author on reasonable request.

\section{Authors' contributions}

YZ and JF conceived and designed the experiments, YZ, JF, $\mathrm{ZF}$ and JL performed the experiments, YZ, JF, ZF and JL analyzed the data and YZ and JF wrote the manuscript. All authors read and approved the final manuscript.

\section{Ethics approval and consent to participate}

Not applicable.

\section{Consent for publication}

Not applicable.

\section{Competing interests}

The authors declare that they have no competing interests.

\section{References}

1. Choudhury RP, Lee JM and Greaves DR: Mechanisms of disease: Macrophage-derived foam cells emerging as therapeutic targets in atherosclerosis. Nat Clin Pract Cardiovasc Med 2: 309-315, 2005.

2. Yuan Y, Li P and Ye J: Lipid homeostasis and the formation of macrophage-derived foam cells in atherosclerosis. Protein Cell 3: 173-181, 2012.

3. Lusis AJ: Atherosclerosis. Nature 407: 233-241, 2000.

4. Berliner JA and Heinecke JW: The role of oxidized lipoproteins in atherogenesis. Free Radic Biol Med 20: 707-727, 1996.

5. Li AC and Glass CK: The macrophage foam cell as a target for therapeutic intervention. Nat Med 8: 1235-1242, 2002.

6. Kleemann R, Zadelaar S and Kooistra T: Cytokines and atherosclerosis: A comprehensive review of studies in mice. Cardiovasc Res 79: 360-376, 2008.

7. Cheng LC, Su KH, Kou YR, Shyue SK, Ching LC, Yu YB, Wu YL, Pan CC and Lee TS: $\alpha$-Lipoic acid ameliorates foam cell formation via liver $\mathrm{X}$ receptor $\alpha$-dependent upregulation of ATP-binding cassette transporters A1 and G1. Free Radic Biol Med 50: 47-54, 2011.

8. Ji A, Meyer JM, Cai L, Akinmusire A, de Beer MC, Webb NR and van der Westhuyzen DR: Scavenger receptor SR-BI in macrophage lipid metabolism. Atherosclerosis 217: 106-112, 2011. 
9. Chen CY, Shyue SK, Ching LC, Su KH, Wu YL, Kou YR, Chiang AN, Pan CC and Lee TS: Wogonin promotes cholesterol efflux by increasing protein phosphatase $2 \mathrm{~B}$-dependent dephosphorylation at ATP-binding cassette transporter-A1 in macrophages. J Nutr Biochem 22: 1015-1021, 2011.

10. Lu KY, Ching LC, Su KH, Yu YB, Kou YR, Hsiao SH, Huang YC, Chen CY, Cheng LC, Pan CC and Lee TS: Erythropoietin suppresses the formation of macrophage foam cells: Role of liver $\mathrm{X}$ receptor alpha. Circulation 121: 1828-1837, 2010.

11. Seo KI, Choi MS, Jung UJ, Kim HJ, Yeo J, Jeon SM and Lee MK: Effect of curcumin supplementation on blood glucose, plasma insulin, and glucose homeostasis related enzyme activities in diabetic db/db mice. Mol Nutr Food Res 52: 995-1004, 2008.

12. Olszanecki R, Jawień J, Gajda M, Mateuszuk L, Gebska A, Korabiowska M, Chłopicki S and Korbut R: Effect of curcumin on atherosclerosis in apoE/LDLR-double knockout mice. J Physiol Pharmacol 56: 627-635, 2005.

13. Zhong Y, Liu T, Lai W, Tan Y, Tian D and Guo Z: Heme oxygenase-1-mediated reactive oxygen species reduction is involved in the inhibitory effect of curcumin on lipopolysaccharide-induced monocyte chemoattractant protein-1 production in RAW264.7 macrophages. Mol Med Rep 7: 242-246, 2013.

14. Youn GS, Kwon DJ, Ju SM, Choi SY and Park J: Curcumin ameliorates TNF- $\alpha$-induced ICAM-1 expression and subsequent THP- 1 adhesiveness via the induction of heme oxygenase- 1 in the HaCaT cells. BMB Rep 46: 410-415, 2013.

15. Kim AN, Jeon WK, Lee JJ and Kim BC: Up-regulation of heme oxygenase-1 expression through CaMKII-ERK1/2-Nrf2 signaling mediates the anti-inflammatory effect of bisdemethoxycurcumin in LPS-stimulated macrophages. Free Radic Biol Med 49: 323-331, 2010.

16. Cremers NA, Lundvig DM, van Dalen SC, Schelbergen RF, van Lent PL, Szarek WA, Regan RF, Carels CE and Wagener FA: Curcumin-induced heme oxygenase- 1 expression prevents $\mathrm{H} 2 \mathrm{O} 2$-induced cell death in wild type and heme oxygenase-2 knockout adipose-derived mesenchymal stem cells. Int J Mol Sci 15: 17974-17999, 2014.

17. Wang S, Zhou H, Feng T, Wu R, Sun X, Guan N, Qu L, Gao Z, Yan J, Xu N, et al: $\beta$-Glucan attenuates inflammatory responses in oxidized LDL-induced THP-1 cells via the p38 MAPK pathway. Nutr Metab Cardiovasc Dis 24: 248-255, 2014

18. Zhong Y, Yu W, Feng J, Fan Z and Li J: Curcumin suppresses tumor necrosis factor- $\alpha$-induced matrix metalloproteinase- 2 expression and activity in rat vascular smooth muscle cells via the NF-кB pathway. Exp Ther Med 7: 1653-1658, 2014.

19. Scapagnini G, Vasto S, Abraham NG, Caruso C, Zella D and Fabio G: Modulation of Nrf2/ARE pathway by food polyphenols: A nutritional neuroprotective strategy for cognitive and neurodegenerative disorders. Mol Neurobiol 44: 192-201, 2011.
20. Rosenson RS, Brewer HB Jr, Davidson WS, Fayad ZA, Fuster V, Goldstein J, Hellerstein M, Jiang XC, Phillips MC, Rader DJ, et al: Cholesterol efflux and atheroprotection: Advancing the concept of reverse cholesterol transport. Circulation 125: 1905-1919, 2012.

21. Wongcharoen $\mathrm{W}$ and Phrommintikul A: The protective role of curcumin in cardiovascular diseases. Int J Cardiol 133: 145-151, 2009.

22. Liu T, Li C, Sun H, Luo T, Tan Y, Tian D and Guo Z: Curcumin inhibits monocyte chemoattractant protein-1 expression and enhances cholesterol efflux by suppressing the c-Jun N-terminal kinase pathway in macrophage. Inflamm Res 63: 841-850, 2014.

23. Zhao JF, Ching LC, Huang YC, Chen CY, Chiang AN, Kou YR, Shyue SK and Lee TS: Molecular mechanism of curcumin on the suppression of cholesterol accumulation in macrophage foam cells and atherosclerosis. Mol Nutr Food Res 56: 691-701, 2012.

24. Takahashi T, Morita K, Akagi R and Sassa S: Heme oxygenase-1: A novel therapeutic target in oxidative tissue injuries. Curr Med Chem 11: 1545-1561, 2004

25. Liu Z, Wang J, Huang E, Gao S, Li H, Lu J, Tian K, Little PJ, Shen X, Xu S and Liu P: Tanshinone IIA suppresses cholesterol accumulation in human macrophages: Role of heme oxygenase-1. J Lipid Res 55: 201-213, 2014

26. Li XY, Wang C, Xiang XR, Chen FC, Yang CM and Wu J: Porphyromonas gingivalis lipopolysaccharide increases lipid accumulation by affecting CD36 and ATP-binding cassette transporter A1 in macrophages. Oncol Rep 30: 1329-1336, 2013

27. Li XY, Kong LX, Li J, He HX and Zhou YD: Kaempferol suppresses lipid accumulation in macrophages through the downregulation of cluster of differentiation 36 and the upregulation of scavenger receptor class B type I and ATP-binding cassette transporters A1 and G1. Int J Mol Med 31: 331-338, 2013.

28. Kim JW, Li MH, Jang JH, Na HK, Song NY, Lee C, Johnson JA and Surh YJ: 15-Deoxy-Delta(12,14)-prostaglandin J(2) rescues $\mathrm{PC} 12$ cells from $\mathrm{H} 2 \mathrm{O} 2$-induced apoptosis through Nrf2-mediated upregulation of heme oxygenase-1: Potential roles of Akt and ERK1/2. Biochem Pharmacol 76: 1577-1589, 2008.

29. Kobayashi M and Yamamoto M: Molecular mechanisms activating the Nrf2-keap1 pathway of antioxidant gene regulation. Antioxid Redox Signal 7: 385-394, 2005.

30. Ma Q, Kinneer K, Bi Y, Chan JY and Kan YW: Induction of murine $\mathrm{NAD}(\mathrm{P}) \mathrm{H}$ :quinone oxidoreductase by 2,3,7,8-tetrachlorodibenzo-p-dioxin requires the CNC (cap ' $n$ ' collar) basic leucine zipper transcription factor Nrf2 (nuclear factor erythroid 2-related factor 2): Cross-interaction between AhR (aryl hydrocarbon receptor) and Nrf2 signal transduction. Biochem J 377: 205-213, 2004. 